\title{
Social Marketing Campaign in Tobacco Control (A Comparative Analysis of Indonesia and Taiwan)
}

\author{
Yeni Rosilawati ${ }^{1 *}$, Chi-Ying Chen ${ }^{2}$, Adhianty Nurjanah $^{1}$, Dianita Sugiyo ${ }^{3,4}$, Shuhui Sophy Cheng ${ }^{5}$ \\ ${ }^{1}$ Department of Communication, Universitas Muhammadiyah Yogyakarta, Yogyakarta, Indonesia; ${ }^{2}$ Department of Information \\ Communication, Asia University, Taichung City, Taiwan; ${ }^{3}$ School of Nursing, Faculty of Medicine and Health Sciences, Universitas \\ Muhammadiyah Yogyakarta, Yogyakarta, Indonesia; ${ }^{4}$ Muhammadiyah Steps, Universitas Muhammadiyah Yogyakarta, \\ Yogyakarta, Indonesia; ${ }^{5}$ Department of Communication Arts, Chaoyang University of Technology, Taichung City, Taiwan
}

Edited by: Sasho Stolesk Citation: Rosilawati Y, Chen C, Nurjanah A, Sugiyo D. Cheng SS. Social Marketing Campaign in Tobacco Contro (A Comparative Analysis of Indonesia and Taiwan). Open Access Maced J Med Sci. 2021 Mar 28; 9(T4):146-150. Keywords: Social marketing; Smoke-free policy; Tobacco use; Health promotion; Tobacco industry *Correspondence: Yeni Rosilawati, Department of Communication, Universitas Muhammadiyah Yogyakarta, Yogyakarta, Indonesia. E-mail: yenirosilawati@gmail.com Received: 07-Feb-202 Revised: 27-Feb-2021 Copyright: ๑ 2021 Yeni Rosilawati, Chi-Ying Chen, hianty Nurianah, Dianita Sugiyo, Shuhui Sophy Chen Funding: This research was supported by Universitas Muhammadiyah Yogyakarta (UMY) and Asia University, Taiwan (No. 107-Asia-UMY-3) Competing Interest: The authors have declared that no Competing interest exist pen Access: This is an open-access article distributed under the terms of the Creative Commons Attribution-

\begin{abstract}
BACKGROUND: Concern about the introduction of young adult smoking is increasing, perhaps because young adults have become a major tobacco marketing target. To solve this issue, both Bali and Taiwan have imposed local regulations on smoke-free areas. Concern about the legalization of young adult smoking is increasing, possibly because young adults have become a major marketing target for tobacco. Both Bali and Taiwan have placed loca restrictions on smoke-free areas to solve this issue.

AIM: This study will analyze the social marketing strategies used to impact young adults in Indonesia and Taiwan focusing specifically on aspects of the social marketing strategy, including channel analysis, market segmentation and consumer analysis, product, price, location and promotion considerations, as well as the implementation and evaluation.

METHODS: This research employed a qualitative approach. Qualitative inquiry is a way of analysis by understanding the meaning of individuals or groups in social problems or human problems. The process comprises questions and procedures. Data collection was conducted by face-to-face interviews. The basic data for this paper came from faceto-face interviews with local government officials, local community leaders and members of the NGO community.

RESULTS: The study found that 4P (Product, Price, Place, and Promotions) with the additional $+2 \mathrm{P}$ (Partnership and Policy) elements of social marketing are employed by social marketing in Bali and Taiwan. The product refers to the principle of a safe life, in which it is easier to avoid than to cure. Price relates to the shift in behavior and the avoidance of early smokers, from smoking to stopping. The distribution networks used in Bali concentrate more on support for families and neighborhoods.

CONCLUSION: The social marketing strategy using 4P (Product, Price, Place, and Promotion) and additional $+2 \mathrm{P}$ (Partnership and Policy) elements is an effective model both in Taiwan and Bali. The commodity on the market is the concept of a safe life, where prevention is better than cure. The price is the shift in action, from smoking to stopping and the prevention of early smoking. The two countries also include other parties, such as schools and community groups, to collaborate.
\end{abstract}

\section{Introduction}

There are literally thousands of studies attesting to the adverse effects of smoking. The longer people smoke, the greater the risks they face. Tobacco, along with more than 4000 other substances that are potentially harmful to health when smoked or chewed, contains nicotine, a highly addictive drug. Indonesia, a nation of 261.1 million people and Southeast Asia's largest economy, is an attractive market for major tobacco companies at a time when demand in more developed markets is slowing. The explanation for the researchers selecting the location between Bali and Taiwan is that there are parallels between the governments of Bali, Indonesia and Taiwan regarding the enforcement of their citizens' no-smoking area policy. The Government of the Province of Bali considers the implementation of the Provincial Regulation on No Smoking Areas
(KTR) in all corners of Bali to be effective in enforcing a no-smoking policy.

The government of Taiwan has also been concerned with the government of Taiwan since September 1, 2019. Offenders shall be charged and fined between NT\$2,000 and NT\$10,000 under the Smoking Risk Prevention Act. The Taipei City Government has released no smoking notices for 2569 seats, including 24 parks and green open spaces, as of July 2019. A community survey was conducted by the Taipei City Government Research, Development and Evaluation Commission in May 2019.

This legislation, generally, speaking, is meant to protect the majority of non-smoking people from the risks of exposure to cigarette smoke. Furthermore, the Regional Control of the KTR also seeks to create a safer community space and atmosphere and to avoid the emergence of inexperienced smokers. KTR is successfully implemented in its application in the field 
of health care services, educational facilities, places where children play, places of worship, public transport hubs, offices, public places, and other places such as sports facilities and city parks. Taiwan, on the other hand, is a South Asian country which is not a member of the World Health Organization and thus cannot be a party to the WHO Tobacco Control Framework Convention.

However, Taiwan has also introduced controlled Smoke Free Places: Where three or more individuals jointly use smoking in indoor workplaces is prohibited; however, this prohibition does not extend to workplaces identified as public places. Smoking in indoor public areas, such as schools, colleges, cultural centers, government offices, and indoor sports and entertainment venues, often has some restrictions. In designated smoking rooms, however, smoking is tolerated in other public places such as hotels, shopping malls, restaurants, cigar shops, bars, and in businesses open only after 9:00 pm and for persons over 18 years of age. This study will analyze the social marketing strategies used to impact young adults in Indonesia and Taiwan focusing specifically on aspects of the social marketing strategy, including channel analysis, market segmentation and consumer analysis, product, price, location and promotion considerations, as well as the implementation and evaluation.

Cigarette smoking has widely been acknowledged as a habit that presents many serious health risks. In particular, numerous regulations, retail protocols, and public health initiatives have been developed to prevent the introduction and uptake of adolescent smoking. According to Canadian estimates, the incidence of smoking among Canadian adolescents decreased more than any other age group between 2001 and 2011 [1].

The prevalence of smoking among those aged $10-18$ years has risen dramatically from $7.2 \%$ in 2013 to $9.1 \%$ in 2018 , according to the country's National Baseline Health Report in 2013 [2] (KEMENKES, 2018). Nevertheless, it was the low-income families who reported an expenditure of $7.2 \%$ of their total tobacco purchasing income that was nominated [3]. The link between teenage smoking and adult addiction was shown in a longitudinal study [4].

It is suggested that adolescents aged 15-17 had 3 times. A study of 5520 American families was surveyed and found that parental smoking is correlated with a high likelihood of adolescent smoking experimentation, as well as transitioning from monthly smoking to regular smoking [1].

Social marketing is the application of commercial marketing strategies, ranging from research, preparation, execution, and assessment of behavioral effect interventions carried out by the public so as to improve the health and welfare status of the population. Social marketing is much the same as marketing concepts in general, except in social marketing, advertisers, and social change practitioners use marketing tactics to support social services or events. Talking about social marketing means talking about campaigns, since the purpose of a campaign is to improve the social behavior of a group to be better than before. In this case, the social marketing aspect or definition does not stand alone but adopts the concept of commercial marketing in general, commonly referred to as a marketing mix or commonly referred to as 4P; which stands for product, price, promotion, and venue. $4 \mathrm{P}$ is a term in commercial marketing that is often used in social marketing, but there is an additional $2 \mathrm{P}$ in social marketing that stands for relationship and regulation [5].

To be more specific about the $4 \mathrm{P}+2 \mathrm{P}$ definition, here is a presentation on the social marketing concept: (1) Product. In social marketing, behavioral change is a type of product offered. However, social media items are not necessarily in physical form. (2) Expense. Price or expense is used to respond to new concepts in actions in the sense of social marketing. Sacrifices related to psychological, mental, social, technological, time, or other welfare aspects can be included in costs or prices [6].

Social marketing prices or losses are incurred to implement new practices by the target group or customers. (3) A spot. Social marketing often wants a place to advertise its social goods, as it does for commercial marketing. (4) Fostering. Promotion defines advertisers' attempts to convey to the target audience improvements in attitudes or ideas so that they are aware of the presence of social goods. (5) Relationship Partnership. There are alliances in social marketing that strive to establish good relationships with different stakeholders, such as government agencies, community organizations, and private companies. This is achieved to generate support for the activities that would be carried out or sold or social goods. (6) The policies. In behavioral improvement processes, social media policies or policies are carried out to inspire the target group [7]. Promoting tobacco and social marketing campaigns through mass media have a beneficial correlation with the initiation of smoking and tobacco use (Freeman, 2012). Initiatives that emphasize tobacco-counter marketing are capable of countering the popular message and eliminating the effects of tobacco marketing strategies [8]. The World Health Organization's Global Regulation on the Elimination of Tobacco Use is known as the 2003 Framework Convention on Tobacco Control [9]. Unlike teen smokers, social marketing influences young adults to smoke and creates a habit of smoking more often or being heavy smokers [10].

Many commercial marketing strategies are used to attract the consumer (audience) to smoking and abstinence by performing segmental research, product marketing mix, and customer enhancement (audience). Long-term action against tobacco marketing campaigns 
should be taken, as the tobacco industry has worked diligently and aggressively for decades to establish and currently sell their products as a standardized tobacco product that is part of the culture of any given group [8].

A comprehensive tobacco counter marketing campaign will only be considered effective if it is integrated, supported by other programs and uses a wide range of techniques and approaches [8]. Moreover, a policy advocacy approach in countering the tobacco industry's messages is important to protect community health through the implementation of smoke free policy that engages closely with community empowerment and public support [11]. Carrying out communication engagement through community health centers (Puskesmas) as the closest health center to the community increasing awareness and promoting understanding amidst the community, to further support the policy implementation and enforcement efforts of the government [11] h;

Tobacco has the same effect as opioids, suggested by the National Institute of Substance Abuse, which results in the release of dopamine that contributes to the reward sensation and pleasure. All these negative tobacco impacts need to be resolved by creating a tobacco-free society that targets the following priorities: (1) stopping people from smoking; (2) harm reduction and quitting programs; (3) safety of tobacco smoke to eliminate second-hand smoke; and (4) denormalization of tobacco products and tobacco industry [12]. There is a close connection between how cigarette companies promote their products and the increasing rate of tobacco prevalence, which is exacerbated by social marketing campaigns using mass media as a medium, which is a significant aspect of convincing people to start or take up smoking and stop smoking (cessation). There is a close connection between how cigarette companies promote their products and the increasing rate of tobacco prevalence, which is exacerbated by social marketing campaigns using mass media as a medium, which is a significant aspect of convincing people to start or take up smoking and stop smoking (cessation) [13].

\section{Methods}

This research employed a qualitative approach. Qualitative inquiry is a way of analysis by understanding the meaning of individuals or groups in social problems or human problems. The process comprised questions and procedures. The basic data for this paper came from face-to-face interviews with local government officials, local community leaders, and members of the NGO community. The data collected were analyzed with an interactive analysis model. This model consists of three analysis components called data reduction, data presentation, and concluding. This research has been approved by the authority and applies informed consent for each respondent with thorough study intent.

\section{Results and Discussion}

\section{Smoke-free policies and regulations}

The non-smoking zone (KTR) laws apply not only in public areas and public facilities. With the ban on cigarette brand banners and even the implementation of cigarette labels, the strict rules of the Klungkung regional government have been improved. Strict laws against cigarette banners or road billboards have also been implemented by the Klungkung local government. It is also necessary for networked shops not to display cigarettes in front of their stores.

While several studies in Indonesia show that smoking among school-aged children is relatively high, the results are varied, likely due to various study designs, methodology, and sample components, rather than because different research models, methods, and sample components. The KTR in Klungkung is used in healthcare, educational facilities, playgrounds, places of worship, public transit hubs, workplaces, and public places. Under these laws, it is predicted that young people and students from all walks of life will not try to smoke, and that those who smoke will be moved to avoid smoking. Although in Taiwan empowerment policy is allocated under the 2009 Tobacco Hazards Prevention Act by expanding smoke-free areas to virtually all enclosed workplaces and public places, introducing graphic health notices to cigarette packets, totally prohibiting tobacco advertising, promotion and sponsorship, and growing tobacco taxes. The Ambitious Tobacco Control Policy implemented in Taiwan in 2009, incorporating smoke-free legislation with a tax increase on tobacco, graphic health warning labels and a total ban on tobacco advertisements, was associated with increases in quit attempt rate and annual cessation rate. Below is an excerpt from the interview with the head of municipal police of Klungkung Regency.

We also promote the detrimental effects of smoking in our weekly and daily meetings with the Banjar (community groups) First, we tell them this at important events, and then we incorporate it into our classroom meetings. It is also not enough to engage with students, but we also need to include parents in this conversation. We do not see their lives outside of school, so we need to remind parents to keep an eye on them.

Meanwhile, in Taiwan, our Informant from the Nantou Public Health Bureau mentioned that:

Our efforts for tobacco prevention have been going on for many years. For school campuses it is 
divided into two parts. The first one is creating a nonsmoking environment, and to do so we have flyers and also inspections. The second part is underage smoking, and for this we have inspectors go onto campuses if the schools are willing. We give parents a letter asking them to keep an eye on their children if we suspect that a student has high chances of smoking. For students that we interview, we also try to find out where they got the cigarettes. Is it in a nearby convenience store, if so, we will go interview that store as well to gather more information. The last part is an increasing reliance on policemen. If they find students who smoke, they will refer the students to us as well.

The informant from Caotun Junior High School added that to help the students to quit smoking is different with the adult's treatment.

Our methods are different, however, because smoking for teenagers is different than smoking for adults. Sometime the teenagers are not actually addicted to tobacco, a lot of times they do it to fit in with their community. In this case, we want to focus on teaching them the right culture, or to help them discover other interest and self-esteem, since a large portion of these smoking teenagers are high school dropouts or have lower grades.

\section{She added:}

It is a cycle that hurts them when they are viewed a certain way by their peers, and when school officials catch them smoking, they have an even worse reputation, causing them to spiral downwards. Thus, our first method is to teach them about cherishing their own lives, and secondly we want them to find hobbies they enjoy doing. We established a community where teenagers who have been helped by us sometimes come back after graduation, and help those newer students in the group. Most times when you directly tell a teenager to quit smoking, they will want to run away from you. But our method of inviting them to play games and build a community seems to be working well in the high school campuses. We have meetings every week and we use that time to educate students on new types of drugs and nicotine products and electronic cigarettes.

In Taiwan, they used teaching materials to educate students on new types of drugs and nicotine products. Moreover, the department of health provides for the programs and the budget. There are four main parts of tobacco prevention, which are teenage tobacco prevention, inspection, promotion of non-smoking environments and services to help quit smoking. In each section there are specialists and project managers to assist planning and implementation, and we also collaborate with some organizations such as the health departments of each county. The Klungkung government hopes that young people and students from all walks of life will not try to smoke and that those who smoke will be encouraged to avoid smoking. KTR is targeted towards public health, where prevention is better than cure. The Klungkung Regency Health Care Program requires rehabilitation that includes the elimination of smoking habits: KBM (Quitting Clinic) entails the participation of Puskesmas and clinics, the procurement of $\mathrm{CO} 2$ equipment and ex-hospitals.

Supporting Institutions and Communities to Bolster the Anti-tobacco Campaigns.

In favor of smoke-free legislation, public health authorities and municipal police have partnered with various organizations such as MTCC Udayana, sponsored by community leaders and organizations such as the Banjar (village), PKK (Family Welfare Program), Posyandu (Integrated Service Post), and religious leaders who support KTR socialization and anti-smoking initiatives. Control of tobacco products cannot only be carried out by the government alone. However, community support and commitment are also needed. One way to do this is by entering into Perarem (local rules) in each village in Klungkung. It is necessary to conduct socialization with community leaders and traditional adat leaders to maintain the nonsmoking area regulation in Klungkung Bali. The policy approach to support the KTR program is a free student transportation program which is a means of tobacco control campaigns, violations of cigarette advertising outside the home, as well as a ban on advertising, promotion and sponsorship of cigarettes in the right places.

The informant from Nantou Public Health Bureau deliberated.

In Taiwan, the tobacco prevention consists of four main parts: Teenage tobacco prevention, inspection, promotion for none-smoking environment, and services to help quit smoking. In each section there are specialists and project managers to assist, and we also collaborate with some organizations such as health departments in each county. As mentioned we divided up to different sections. For example, doctors in Nantou would have their own alliance group, and different counties would also have their own groups. There are two reasons for these alliances. First, we want everyone to know each other, and the second is that we want clearer communication when we need help.

\section{She added:}

We actually have a lot of collaborations. We have teachers because they are impactful for youths, and we also have the Police Department as well as hospitals. We work with pharmacist's unions and also doctor's unions because we want to utilize their professional skills. We also work with offices to create non-smoking workplaces. For school campuses it is divided into two parts. The first one is creating a nonsmoking environment, and to do so we have flyers and also inspections. The second part is underage smoking, and for this we have inspectors go onto campuses if the schools are willing. We give parents a letter asking 
them to keep an eye on their children if we suspect that a student has high chances of smoking. For students that we interview, we also try to find out where they got the cigarettes. Is it is in a nearby convenience store, we will go interview that store as well to gather more information. the last part we rely on policemen. If they find students who smoke, they will refer the students to us as well.

In Taiwan, the schools had implemented weekly and daily meetings to inform students on the negative effects of smoking and conduct some of event to get the students attracted to non-smoking. This is in line that a policy advocacy approach in countering the tobacco industry's messages is important to protect community health through the implementation of smoke free policy that engages closely with community empowerment and public support [11].

In Taiwan, schools held daily and weekly meetings to educate students about the dangers of smoking. They attract the student's attendance by first explaining the meeting goals and benefits garnered. Specific strategies have been implemented, including setting up physical education classes and health classes, which are programs fully supported by the Department of Health. Taiwan also supported by an alliance of doctors, teachers, and Pharmacists unions.

The school in collaboration with the health department also conducted yearly carbon monoxide analysis for all the students, to paint a portrait of passive and active smokers, especially for those passive smokers who are affected in their household environment. The media then promoted the campaigns through advocacy in schools, communities, posters and audio visual forms (film). The campaign promoted partnerships and collaborations with other community organizations and also some institutions with a similar mission against Tobacco.

Social media and health promotion methods have been implemented in both countries to warn people about the risks of cigarettes and to apply a free smoking zone. The Klungkung Government hopes that young people and students from all walks of life will not try to smoke, and that those who smoke will be encouraged to avoid smoking. KTR is targeted toward public health, where prevention is better than cure. Tobacco prevention focuses in Taiwan on four main areas: Prevention of adolescent tobacco, inspection, promotion. The study found that social marketing using 4P (Product, Price, Place, and Promotion) and additional $+2 \mathrm{P}$ (Partnership and Policy) elements is an effective model both in Taiwan and Bali. The commodity on the market is the concept of a safe life, where prevention is better than cure. The price is the shift in action, from smoking to stopping, and the prevention of early smoking. The two countries also include other parties, such as schools and community groups, to collaborate. In Taiwan, the schools held meetings to educate students about the harmful effects of smoking and to arrange some event to attract non-smoking students. Taiwan also supported the alliance of physicians, teachers and pharmacists' unions as their stakeholders and engaged them in a variety of activities. Social marketing can be successful when socialization, implementation, oversight, and compliance of the relevant regulations are carried out as concrete measures.

\section{Conclusion}

The social marketing strategy using 4P (Product, Price, Place, and Promotion) and additional $+2 \mathrm{P}$ (Partnership and Policy) elements is an effective model both in Taiwan and Bali.

\section{References}

1. Janz T. Health at a Glance: Current Smoking Trends: 2012. Available from: http://www.statcan.gc.ca/pub/82624-x/2012001/article/11676-eng.htm. [Last accessed on 2020 Jul 02].

2. Kemenkes. Hasil Utama Riskesdas. Indonesia: Kementerian Kesehatan Republik Indonesia; 2018

3. World Health Organisation.Global Youth Tobacco Survey (GYTS) Indonesia Report 2014. Geneva: World Health Organisation; 2015.

4. Griffin KW, Botvin GJ, Doyle MM, Diaz T, Epstein JA. A six-year follow-up study of determinants of heavy cigarette smoking among high-school seniors. J Behav Med 1999;22:271-84. PMid:10422618

5. Andreasen A. Social marketing: "Its definition and domain". J Market Public Policy 1994;13:108-14.

6. Gallopel-Morvan K. The use of visual warnings in social marketing: The case of tobacco. J Bus Res 2011;64:7-11.

7. McLean G. Social Marketing. SBS HDR Student Conference; 2010. Available from: http://www.ro.uow.edu.au/sbshdr/2010/ papers. [Last accessed on 2020 Jul 02].

8. Kotler P, Gertner D. Theoretical Papers. Country as Brand, Product, and Beyound: A Place Marketing and Brand Management Perspective. Special Issue Brand Management 2002. p. 4-5. https://doi.org/10.1057/palgrave.bm. 2540076

9. Centers for Disease Control and Prevention. Designing and Implementing an Effective Tobacco Counter-Marketing Campaign. California, Amerika: CreateSpace Independent Publishing Platform; 2014

10. Hayward LI. Social Marketing to Influence Young Adults. Tobacco Behaviour. Thousand Oaks, California: SAGE; 2012.

11. Nurjanah A, Sugiyo D, Mutiarin D. Health communication to support policy advocacy of non-smoking area (KTR) in the special region of Yogyakarta, Indonesia. Int Med J 2020;25:845-50.

12. The British Medical Association. Promoting a Tobacco-free Society. United Kingdom: The BMA Board of Science; 2015.

13. Freeman B. New media and tobacco control. Tobacco Control 2012;21:139-44. 